\title{
DETERMINACIÓN Y CUANTIFICACIÓN DE MERCURIO TOTAL EN TEJIDO MUSCULAR DE PARGO (LUTJANUS COLORADO), MEDIANTE LA TÉCNICA DE VAPOR FRÍO ACOPLADO A ESPECTROFOTOMETRÍA DE FLUORESCENCIA ATÓMICA
}

\section{DETERMINATION AND QUANTIFICATION OF TOTAL MERCURY IN MUSCULAR TISSUE OF SNAPPER (LUTJANUS COLORADO), USING THE TECHNIQUE OF COLD STEAM COUPLED TO ATOMIC FLUORESCENCE SPECTROPHOTOMETRY}

Leonardo Bustamante1 ${ }^{1}$ Lenys Fernández ${ }^{1} \&$ Gabriela Cueva T. ${ }^{*}$

Recibido: 9 de noviembre 2020 / Aceptado: 17 de diciembre 2020 DOI: 10.26807/ia.v9i1.201

Palabras claves: Fluorescencia atómica, mercurio total, pez pargo, vapor frío. Key words: Atomic fluorescence, cold vapor, pargo fish, total mercury.

1 Pontificia Universidad Católica del Ecuador, Facultad de Ciencias Exactas y Naturales, Escuela de Ciencias Químicas, Quito Ecuador (leonardobg18@hotmail.com, Imfernandez@puce.edu.ec, *correspondencia: gfcueva@puce.edu.ec, gfcueva@gmail.com 


\section{RESUMEN}

Se determinó mercurio total en pescado pargo mediante la técnica de vapor frío acoplado a espectrofotometría de fluorescencia atómica (CV-AFS, por sus siglas en inglés). Se llevó a cabo un muestreo no probabilístico en el mercado América de la ciudad de Quito. Las muestras de pescado se sometieron a un proceso de liofilización y digestión ácida asistida por microondas. Se obtuvo valores de concentración de mercurio total de 0,077;0,079;0,046;0,093 y $0,113 \mathrm{mg} \mathrm{kg}^{-1}$ respectivamente, cuyos coeficientes de variación fueron 5,00; 3,$91 ; 3,01 ; 16,1$ y $14,5 \%$. Para las muestras de pargo mediano se obtuvieron valores de 0,$163 ; 0,111 ; 0,176 ; 0,201$ y $0,185 \mathrm{mg} \mathrm{kg}^{-1}$, y sus coeficientes de variación fueron 13,$3 ; 17,6 ; 8,18 ; 9,64$ y $14,5 \%$. Mientras que para las muestras de pargo grande los valores obtenidos fueron: 0,$160 ; 0,182 ; 0,176 ; 0,361$ y $0,170 \mathrm{mg} \mathrm{kg}^{-1}$, dando coeficientes de variación de 10,6; 8,79; 16,8; 4,57 y $9,45 \%$. Las muestras de pargo grande presentaron un mayor contenido de mercurio en su organismo. Se determinó que las muestras de pescado contienen mercurio, sin embargo, no exceden los límites máximos permisibles establecidos por el Codex Alimentarius.

\section{ABSTRACT}

The determination of total mercury in fish allows estimating what concentration of this metal is present in these aquatic organisms. Total mercury was determined in 45 samples of snapper fish by means of the cold vapor technique coupled to atomic fluorescence spectrophotometry. A non-probabilistic sampling was carried out in the América market in Quito. The fish samples were subjected to a microwave-assisted acid digestion and lyophilization process. In the experimental results, total mercury concentration values of 0.077 were obtained; $0.079 ; 0.046 ; 0.093$ and $0.113 \mathrm{mg} \mathrm{kg}^{-1}$ respectively, whose coefficients of variation were $5.00 ; 3.91 ; 3.01 ; 16.1$ and $14.5 \%$. For the medium snapper samples, values of 0.163 were obtained; $0.111 ; 0.176 ; 0.201$ and $0.185 \mathrm{mg} \mathrm{kg}^{-1}$, and their coefficients of variation were $13.3 ; 17.6 ; 8.18 ; 9.64$ and $14.5 \%$. While for the large snapper samples the values obtained were: $0.160 ; 0.182$; 
$0.176 ; 0.361$ and $0.170 \mathrm{mg} \mathrm{kg}^{-1}$, giving coefficients of variation of $10.6 ; 8.79$; 16.8; 4.57 and $9.45 \%$. The large snapper samples had a higher mercury content in their body. It was determined that the fish samples contain total mercury, however, they do not exceed the maximum permissible limits established by the Codex Alimentarius.

\section{INTRODUCCIÓN}

Los ríos y océanos son propensos a la contaminación de metales pesados ocasionada por fuentes naturales y antropogénicas, sobre todo por industrias que desechan múltiples materiales con metales pesados entre los que se encuentra el mercurio (Reyes et al., 2016). Por lo anterior, es necesario determinar si las especies acuáticas como los peces y en particular los considerados altamente comestibles, como el pargo (Lutjanus colorado), están siendo afectados por la presencia y cantidad de este metal, y sobre todo si al ser consumidos por el ser humano éste lo está asimilando en su organismo.

El pescado es un alimento que posee proteínas de alto valor biológico y ácidos grasos esenciales, de allí su alto consumo (Müller et al., 2014).
Los peces son parte del grupo de alimentos que pueden acumular en su organismo grandes cantidades de metales traza como el mercurio y el selenio (Bergés et al., 2015). El mercurio $(\mathrm{Hg})$ es un contaminante persistente de elevada toxicidad para los seres vivos, lo que aumenta el riesgo en el ámbito de la salud humana, ya que el ingreso de este metal al medio ambiente facilita su acumulación en la cadena alimenticia Ilegando de esta manera a las personas por medio del consumo del pescado (Moraes et al., 2013).

El mercurio orgánico se encuentra en el agua, principalmente como metilmercurio $\left[\mathrm{CH}_{3} \mathrm{Hg}\right]^{+}$y es el componente más común en la cadena alimentaria (Rezende, Silva, Moura, \& Windmöller, 2018). 
La espectrometría de fluorescencia atómica (AFS, por sus siglas en inglés) se ha convertido en una de las herramientas analíticas más importantes para el análisis de oligoelementos incluido el mercurio (Krishna et al., 2005). La detección de Hg por esta técnica acoplada a la técnica de vapor frío (CV, por sus siglas en inglés), como técnica de introducción de muestra, está reemplazando a la espectroscopia de absorción atómica (AAS, por su siglas en inglés) en el análisis de este metal, debido a su instrumentación simple y límites de detección ultra bajos, alta sensibilidad, selectividad, fácil y bajo consumo de gas argón (Aranda, Gil, Moyano, De Vito \& Martinez, 2009); convirtiéndose en una de las herramientas analíticas más importantes para el análisis de oligoelementos (Krishna et al., 2005). En el presente trabajo se reporta la detección y cuantificación de Hg por CV- AFS, a fin de obtener un acercamiento sobre el contenido de este metal en los peces que comúnmente está consumiendo la comunidad citadina de Quito-Ecuador (Chaves, 2016).

\section{MATERIALES Y MÉTODOS}

\section{Toma de muestra}

Para este trabajo diagnóstico, la toma de muestras de pescado pargo se realizó en el mercado popular de abastos "América" del Distrito Metropolitano de Quito (Pichincha - Ecuador), mediante muestreo no probabilístico por conveniencia, basado en la selección de una muestra de la población por el hecho de ser accesible (Ochoa, 2015).

La muestra se recolectó y tipificó en cinco por cada tamaño, pequeño, mediano y grande, y cada pescado se analizó por triplicado, dando un total de 45 muestras analizadas. Los pescados se transportaron al laboratorio bajo cadena de frío a una temperatura aproximada de $4{ }^{\circ} \mathrm{C}$, manteniéndola hasta su posterior preparación y tratamiento (EPA, 2000).

\section{Preparación de muestra}

Se realizó la medición de los pescados, determinando la longitud total 
(largo del pescado) y la longitud estándar que excluye el largo de la aleta caudal (Segura et al., 2011). Se determinaron los rangos de medición conforme a los datos obtenidos de la longitud estándar de cada pez, dando como resultado $(25,0-26,5 \mathrm{~cm})$, $(35,0-43,0 \mathrm{~cm})$ y $(43,5-50,5 \mathrm{~cm})$ para las muestras de pargo pequeño, mediano y grande respectivamente.

Posteriormente, se obtuvieron submuestras del músculo dorsal de cada pez y se realizó la determinación de humedad mediante la balanza infrarroja HB43-S, marca Mettler Toledo, Suiza, la cual utiliza un sistema de análisis termogravimétrico. Para la medición de humedad se colocó la muestra del músculo de pargo en el plato de la balanza en un rango de 2,6-3,5 g, donde se secó a una temperatura de $160{ }^{\circ} \mathrm{C}$ por un periodo aproximado de 40 minutos.

La muestra se sometió a un proceso de ultracongelación a $-25{ }^{\circ} \mathrm{C}$ en la ultracongeladora marca Infrico Medcare, España; y luego se liofilizó por un tiempo aproximado de 48 horas en un equipo Cool Safe Touch 55-4, marca Labogene, Dinamarca. Una vez liofilizada la muestra, se pulverizó y se separó en submuestras de 1 $\mathrm{g}$ en bolsas herméticas y debidamente etiquetadas dentro de un desecador.

\section{Digestión ácida asistida por mi- croondas}

Las muestras de pescado liofilizadas se digirieron en un equipo microondas MARS 6, marca CEM, EUA. Se trabajó en viales de teflón para digestión de alta presión, MARS EasyPrep.

Para la digestión de muestra de pescado se adecuó el método propuesto por Shah et al. (2012), utilizando 0,3 g de muestra liofilizada. Posteriormente, se añadió en el vial de teflón $1 \mathrm{~mL}$ de ácido nítrico concentrado $\left(\mathrm{HNO}_{3}\right), 1 \mathrm{~mL}$ de peróxido de hidrógeno concentrado $\left(\mathrm{H}_{2} \mathrm{O}_{2}\right)$ y $1 \mathrm{~mL}$ de ácido perclórico concentrado $\left(\mathrm{HClO}_{4}\right)$. A continuación, se acondicionó el programa de digestión. En la Tabla 1 se presentan las condiciones utilizadas para esta digestión: 
Tabla 1. Condiciones del programa de digestión en microondas

\begin{tabular}{cccccc}
\hline Paso & \multicolumn{2}{c}{ Potencia } & \begin{tabular}{c} 
Rampa \\
de tiempo \\
\cline { 2 - 4 }$(\min )$
\end{tabular} & $\begin{array}{c}\text { Temperatura } \\
\left({ }^{\circ} \mathrm{C}\right)\end{array}$ & $\begin{array}{c}\text { Tiempo de } \\
\text { retención } \\
(\text { min) }\end{array}$ \\
\hline 1 & $1400 \mathrm{~W}$ & 80 & 20 & 210 & 15 \\
\hline
\end{tabular}

Posterior a la digestión por microondas, las muestras se filtraron y se añadió $1 \mathrm{~mL}$ de ácido clorhídrico concentrado $(\mathrm{HCl})$. Luego se aforó en matraces aforados de $50 \mathrm{~mL}$ con agua grado reactivo de alta pureza (resistividad $18,2 \mathrm{M} \Omega \mathrm{cm}$ a $25^{\circ} \mathrm{C}$ ) obtenida de un sistema de agua pura directa Genie 5 Direct-Pure Water System marca Rephile, China. A continuación, se trasvasaron las soluciones a los viales de inyección para realizar el análisis en el equipo de CV-AFS.

Cuantificación de mercurio mediante la técnica de vapor frío acoplado a espectrofotometría de fluorescencia atómica

La mejor señal de absorbancia se determinó mediante un diseño experimental completamente al azar, ya que las unidades experimentales fueron homogéneas y es un método flexible en cuanto al número de repeticiones. Los niveles fueron las relaciones de concentración (Figura 1) y la variable de respuesta fue la intensidad de absorbancia de señal obtenida.

Para la cuantificación de mercurio total mediante CV-AFS, se utilizó el equipo Mercur-Plus, marca Analytik Jena, Alemania. Para la operación del equipo se utilizó ácido clorhídrico al 2 \% para blancos, solución ácida de reacción, soluciones de dilución y de enjuague, y cloruro de estaño al $2 \%$ preparado en ácido clorhídrico al $4 \%$, para la reacción de reducción.

Se preparó una solución madre de mercurio de $50 \mu \mathrm{g} \mathrm{L}^{-1}$ a partir del estándar de mercurio $(9,995 \pm 0.056$ $\mathrm{mg} \mathrm{L}^{-1}$ ), Inorganic Ventures, Estados Unidos. El equipo se programó para diluir esta solución e inyectar automáticamente para la construcción de una curva de calibración de 1, 2, 3, 4 y $5 \mu \mathrm{g} \mathrm{L}^{-1}$. Todas las soluciones fue- 
ron preparadas en agua grado reactivo calidad alta pureza.

La reacción de generación de vapor se dio empleando $\mathrm{SnCl}_{2}$ en medio ácido como agente reductor (Pohl et al., 2008). Las muestras se analizaron por triplicado y las lecturas por duplicado. Además, por cada lote de 11 muestras se leyó un material de referencia certificado (MRC) DORM-4 fish protein, National Research Council Canadá y un blanco. Finalmente, se programó el software para la inyección automática de las muestras previamente digestadas en microondas.

\section{RESULTADOS}

En las Tablas 2, 3 y 4 se detalla el contenido de humedad de las muestras, se reporta el peso de cada una y el promedio total de las cinco diferentes muestras de pescado pargo, por tamaños. A cada pescado se le asignó un código respectivo para su identificación, donde se indica el nombre de la especie (PG), el número de muestra (001) y el tamaño del pescado (p). Como se ve en la Tabla 2 se determinó el peso de las muestras de pargo pequeño cuyo valor promedio fue de $262 \mathrm{~g}$, y el porcentaje de humedad fue de 78,10 $\%$.

Tabla 2. Contenido de humedad de 5 muestras de pargo pequeño (Lutjanus colorado)

\begin{tabular}{ccc}
\hline Código asignado & Peso $(\mathrm{g})$ & Humedad (\%) \\
\hline Pg001p & 273,6 & 79,32 \\
Pg002p & 262,6 & 79,53 \\
Pg003p & 282,0 & 77,05 \\
Pg004p & 231,8 & 77,79 \\
Pg005p & 259,9 & 76,83 \\
Promedio & 261,9 & 78,10 \\
\hline
\end{tabular}


En la Tabla 3 se observa que las muestras de pescado mediano presentaron un peso con un valor promedio aproximado de 891,2 g y un porcentaje de humedad de aproximadamente $76,68 \%$.

Tabla 3. Contenido de humedad de cinco muestras de pargo mediano (Lutjanus colorado)

\begin{tabular}{ccc}
\hline $\begin{array}{c}\text { Código } \\
\text { asignado }\end{array}$ & $\begin{array}{c}\text { Peso } \\
(\mathrm{g})\end{array}$ & $\begin{array}{c}\text { Humedad } \\
(\%)\end{array}$ \\
\hline Pg006m & 726,1 & 75,75 \\
Pg007m & 641,5 & 75,80 \\
Pg008m & 1095 & 76,95 \\
Pg009m & 1116 & 77,22 \\
Pg010m & 876,7 & 77,68 \\
\hline Promedio & 891,2 & 76,68 \\
\hline
\end{tabular}

En la Tabla 4 se detallan los datos obtenidos del espécimen con mayor tamaño. El porcentaje promedio de las muestras de pargo grande dio un valor de 76,38\%, siendo el valor más bajo obtenido en el análisis. Por otro lado, las muestras que tuvieron un mayor porcentaje de humedad fueron las de pargo pequeño con un valor aproximado de 78,10 \%.
Tabla 4. Contenido de humedad de cinco muestras de pargo grande (Lutjanus colorado)

\begin{tabular}{ccc}
\hline $\begin{array}{c}\text { Código } \\
\text { asignado }\end{array}$ & $\begin{array}{c}\text { Peso } \\
(\mathrm{g})\end{array}$ & $\begin{array}{c}\text { Humedad } \\
(\%)\end{array}$ \\
\hline Pg006m & 1070 & 77,78 \\
Pg007m & 1619 & 76,00 \\
Pg008m & 1356 & 76,66 \\
Pg009m & 1726 & 74,58 \\
Pg010m & 1352 & 76,87 \\
\hline Promedio & 1425 & 76,38 \\
\hline
\end{tabular}

Para determinar la concentración de mercurio total en cada una de las muestras se elaboró una curva de calibración multipunto, Figura 1, a concentraciones 1, 2, 3, 4 y $5 \mu \mathrm{g} \mathrm{L}^{-1}$, que fueron diluidas por el inyector automático utilizando la solución estándar de $50 \mu \mathrm{g} \mathrm{dm}{ }^{-3}$ de $\mathrm{Hg}$.

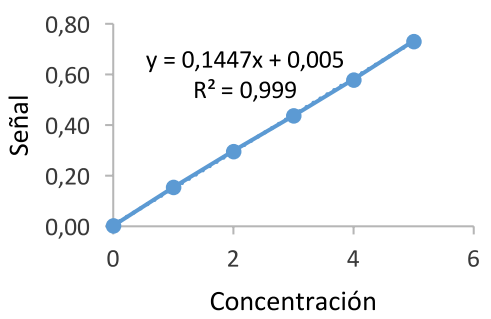

Figura 1. Curva de calibración obtenida en el equipo de CV-AFS

Como se muestra en la Figura 1, la relación señal-concentración es direc- 
tamente proporcional. En otras palabras, la intensidad de emisión de energía (fluorescencia) se va a dar en función de la cantidad de átomos del analito que se encuentren en estado excitado.
Como se puede evidenciar en la Tabla 5, los resultados obtenidos en la curva de calibración garantizan un ajuste lineal positivo, considerando el coeficiente de regresión lineal $\left(\mathrm{R}^{2}>\right.$ $0,999)$. Esto demuestra que los valores obtenidos son aceptables.

Tabla 5. Datos obtenidos de la curva de calibración

\begin{tabular}{ccc}
\hline $\begin{array}{c}\text { Coeficiente } \\
\text { de correlación }\end{array}$ & Pendiente & Intercepto \\
\hline 0,9999 & 0,1447 & 0,005 \\
\hline
\end{tabular}

Tabla 6. Porcentajes de recuperación obtenidos del material de referencia certificado (MRC)

\begin{tabular}{lcccccc}
\hline Muestra & $\begin{array}{c}\text { Peso } \\
(\mathrm{g})\end{array}$ & $\begin{array}{c}\text { Lectura 1 } \\
\left(\mu \mathrm{g} . \mathrm{L}^{-1}\right)\end{array}$ & $\begin{array}{c}\text { Promedio } \\
\text { Lecturas } \\
\left(\mu \mathrm{g} \cdot \mathrm{L}^{-1}\right)\end{array}$ & $\begin{array}{c}\text { Resultado } \\
\text { Blanco } \\
\left(\mu \mathrm{g} \cdot \mathrm{L}^{-1}\right)\end{array}$ & $\begin{array}{c}\text { Resultado } \\
1 \text { peso seco } \\
\left(\mu \mathrm{kg} \mathrm{kg}^{-1}\right)\end{array}$ & $\%$ R \\
\hline \multirow{4}{*}{ MRC } & 0,296 & 2,421 & 2,421 & 0,109 & 390,1 & 95 \\
& 0,293 & 2,350 & 2,350 & 0,051 & 391,1 & 95 \\
& 0,297 & 2,500 & 2,500 & 0,073 & 409,3 & 99 \\
& 0,306 & 2,567 & 2,567 & 0,073 & 407,1 & 99 \\
\hline
\end{tabular}

*Volumen de aforo es de $50 \mathrm{~mL}$

La exactitud de la metodología se estableció analizando un material de referencia certificado CRM DORM-4. Esto en base a la recuperación de mercurio total ( $\mathrm{T}-\mathrm{Hg}$ ) calculada de acuerdo con el valor certificado (MRC) que es de $412 \pm 36 \mu \mathrm{gg}^{-1}$. El porcentaje de recuperación obtenido fue del 95-99\%, como se ve en la Tabla 6. Los porcentajes de recuperación están dentro del rango de aceptación determinado por el Codex Alimentarius (80-120\%). Por tanto, el método propuesto muestra la exactitud necesaria para analizar mercurio en el pescado. 


\section{Concentraciones de mercurio}

A continuación se detallan las concentraciones de mercurio total obtenidas en las muestras de pargo (Lutjanus colorado) que se expenden en el mercado América de la ciudad de Quito (Tabla 7). Se obtuvieron valores de mercurio total en peso seco de $199,4 \mu \mathrm{g} \mathrm{kg}^{-1} \pm 0,70$ hasta 486,4 $\mu \mathrm{g} \mathrm{kg}{ }^{-1} \pm 3,37$; mientras que la concentración en peso húmedo fue entre $45,76 \mu \mathrm{g} \mathrm{kg}^{-1} \pm 3,03$ hasta $112,7 \mu \mathrm{g}$ $\mathrm{kg}^{-1} \pm 14,6$. Finalmente, se obtuvieron valores entre $0,046 \mathrm{mg} \mathrm{kg}^{-1} \pm$ 3,01 hasta $0,113 \mathrm{mg} \mathrm{kg}^{-1} \pm 14,5 \mathrm{de}$ mercurio total en peso húmedo o peso fresco de pescado pequeño.

\section{Tabla 7. Concentraciones de mercurio total obtenidas en las muestras de pargo pequeño}

\begin{tabular}{ccccc}
\hline & $\begin{array}{c}\text { Promedio } \\
\left(\mu_{\text {gg.kg }}{ }^{-1}\right)\end{array}$ & $\%$ CV & $\begin{array}{c}\text { Peso } \\
\text { (Húmedo) }\end{array}$ & $\begin{array}{c}\text { Peso } \\
\text { (Seco) }\end{array}$ \\
\hline Pg001 & 373,5 & 1,03 & 77,24 & 0,077 \\
Pg002 & 386,6 & 0,80 & 79,13 & 0,079 \\
Pg003 & 199,4 & 0,70 & 45,76 & 0,046 \\
Pg004 & 419,2 & 3,58 & 93,10 & 0,093 \\
Pg005 & 486,4 & 3,37 & 112,7 & 0,113 \\
\hline
\end{tabular}

En la Tabla 8 se observa que los valores de concentración de $\mathrm{T}-\mathrm{Hg}$ en peso seco fueron entre 460,2 $\mu \mathrm{kg}^{-1} \pm$ 4,24 hasta 881,2 $\mathrm{ug} \mathrm{kg}^{-1} \pm 2,19$, mientras que la concentración en peso húmedo fue entre $111,4 \mu \mathrm{g} \mathrm{kg}{ }^{-1}$ $\pm 17,5$ hasta $200,7 \pm 9,65$. Finalmente, se obtuvieron valores entre $0,111 \mathrm{mg} \mathrm{kg}^{-1} \pm 17,6$ hasta $0,201 \mathrm{mg}$ $\mathrm{kg}^{-1} \pm 9,64$ de mercurio total en peso húmedo o peso fresco de pescado mediano. 
Tabla 8. Concentraciones de mercurio total obtenidas en las muestras de pargo mediano

\begin{tabular}{ccccc}
\hline & $\begin{array}{c}\text { Promedio } \\
\left(\mu \mathrm{g} \mathrm{kg}{ }^{-1}\right)\end{array}$ & $\% \mathrm{CV}$ & $\begin{array}{c}\text { Peso } \\
\text { (Húmedo) }\end{array}$ & $\begin{array}{c}\text { Peso } \\
(\text { Seco) }\end{array}$ \\
\hline Pg006 & 672,8 & 3,23 & 162,9 & 0,163 \\
Pg007 & 460,2 & 4,24 & 111,4 & 0,111 \\
Pg008 & 762,1 & 1,88 & 175,7 & 0,176 \\
Pg009 & 881,2 & 2,19 & 200,7 & 0,201 \\
Pg010 & 828,9 & 3,24 & 185,0 & 0,185 \\
\hline
\end{tabular}

En la Tabla 9 se reportan concentraciones de T-Hg entre $719,6 \mu \mathrm{g} \mathrm{kg}^{-1} \pm$ 2,35 hasta $1419 \mu \mathrm{g} \mathrm{kg}^{-1} \pm 1,16$, mientras que la concentración en peso húmedo fue entre $159,9 \mu \mathrm{g} \mathrm{kg}^{-1} \pm 10,6$ hasta $360,7 \pm 4,6$. Finalmente, se obtuvieron valores entre $0,160 \mathrm{mg} \mathrm{kg}^{-1}$ $\pm 10,6$ hasta $0,361 \mathrm{mg} \mathrm{kg}^{-1} \pm 4,57 \mathrm{de}$ mercurio total en peso húmedo o peso fresco de pescado grande. Las muestras de pescado pargo grande presentaron un mayor contenido de mercurio en su organismo según los datos obtenidos en el presente estudio.

\section{Tabla 9. Concentraciones de mercurio total obtenidas en las muestras de pargo grande}

\begin{tabular}{ccccc}
\hline & $\begin{array}{c}\text { Promedio } \\
\left(\mu \mathrm{kg}^{-1}\right)\end{array}$ & \% CV & $\begin{array}{c}\text { Peso } \\
\text { (Húmedo) }\end{array}$ & $\begin{array}{c}\text { Peso } \\
\text { (Seco) }\end{array}$ \\
\hline Pg011 & 719,6 & 2,35 & 159,9 & 0,160 \\
Pg012 & 756,5 & 2,12 & 181,6 & 0,182 \\
Pg013 & 753,0 & 3,92 & 175,8 & 0,176 \\
Pg014 & 1419 & 1,16 & 360,7 & 0,361 \\
Pg015 & 735,3 & 2,18 & 170,1 & 0,170 \\
\hline
\end{tabular}

Tanto el límite de detección (LD) como el límite de cuantificación (LC) se determinaron mediante la medi- ción de blancos. Se prepararon 47 muestras blanco de ácido clorhídrico al $2 \%$ y se realizaron las respectivas 
lecturas aplicando el método de estudio. El LD se determinó conociendo el promedio de la señal del blanco más tres veces su desviación estándar $(y+3 S)$; y el LC se calculó conociendo el promedio de la señal del blanco más 10 veces su desviación estándar $(y+10 S)$. Dichos valores se muestran en la Tabla 10.
Tabla 10. Límites de detedetección y cuantificación obtenidos en el presente estudio

\begin{tabular}{cc}
\hline LD $\left(\mu \mathrm{g} \mathrm{L}^{-1}\right)$ & LC $\left(\mu \mathrm{L}^{-1}\right)$ \\
\hline 0,111 & 0,237 \\
\hline
\end{tabular}

\section{DISCUSIÓN}

\section{Concentración de mercurio}

De acuerdo con el Codex Alimentarius, se aclara que el metilmercurio constituye la mayor parte del mercurio total en la mayoría de las especies marinas. El metilmercurio es una de las formas más tóxicas que presenta el mercurio y puede llegar a conformar entre el $80-100 \%$ del mercurio total que está presente en los peces (González et al., 2015).

La concentración de mercurio depende de la afinidad que posee con el músculo de pescado. La acumulación de mercurio se debe precisamente al metilmercurio, el cual se adsorbe y acumula más que otras formas en el músculo de pescado (González et al., 2014). Los peces fijan con fuerza al metilmercurio, el cual equivale casi al $100 \%$ del mercurio que se bioacumula, por lo que la mayor parte del metilmercurio en tejidos de peces forma enlaces covalentes con grupos sulfidrilo proteínico, con lo que la vida media de eliminación resulta larga (De Carvalho et al., 2010).

La cantidad de mercurio en la muestra, además, va a depender de otros factores como la localidad en la cual puede estar en contacto o disponibilidad hacia ella; ya que las concentraciones de tipo de metal van a ser mayores en ciertos lugares geográficos que están expuestos a una mayor contaminación, ya sea por la eliminación de desechos causado por las industrias o por procesos biológicos naturales (metilación). Aunado a lo 
anterior, está la disponibilidad de alimentos (otros organismos acuáticos) y el contenido de mercurio que presenten (biomagnificación), ya que las especies más proclives a la acumulación de mercurio son las especies depredadoras que se encuentran en el nivel más alto de la cadena trófica como albacora, atún, pez espada, tiburón, entre otros. Por tanto, van a tener una mayor cantidad de mercurio (metilmercurio) en su organismo. Además, hay otros factores que también podrían influir como el género, el tamaño, la edad, etc. (González et al., 2014).
En la literatura no existen los límites máximos permisibles con relación a la cantidad de mercurio total que existe en el pez pargo (Lutjanus colorado). Sin embargo, de acuerdo con el Codex Alimentarius, se ha establecido que el contenido máximo de metilmercurio en la carne de pescado debe ser de 0,5 $\mathrm{mg} \mathrm{kg}^{-1}$ en peso fresco para los productos de pesca incluidos los mariscos (Comisión del Codex Alimentarius, 2018). Los resultados obtenidos en el presente trabajo indican que ninguna de las muestras de pargo analizadas excede los límites máximos permisibles establecidos sobre el mercurio en el pescado.

\section{CONCLUSIÓN}

El porcentaje de humedad obtenido en las muestras de Pargo de tamaños pequeño, mediano y grande dio un valor promedio de 78,10; 76,68 y $76,38 \%$ respectivamente.

Se determinó que todas las muestras de pescado presentan mercurio total en su organismo, sin embargo, estos valores no exceden los límites permitidos por el Codex Alimentarius que es de $0,5 \mathrm{mg} \mathrm{kg}^{-1}$ de mercurio.
Con respecto a la técnica CV-AFS, es una técnica selectiva para el análisis de mercurio total en el pescado, ya que éste es el único metal que puede ser analizado mediante el sistema de vapor frío, por ser líquido a temperatura ambiente.

Además, se obtuvo un límite de detección de $0,111 \mu \mathrm{g} \mathrm{L}^{-1}$, mientras que el límite de cuantificación fue de $0,237 \mu \mathrm{g} \mathrm{L^{-1 }}$, lo que demuestra que la técnica CV- AFS es más sensible en comparación a las técnicas de AAS. 


\section{LISTA DE REFERENCIAS}

Aranda, P. R., Gil, R. A., Moyano, S., De Vito, I., \& Martinez, L. D. (2009). Slurry sampling in serum blood for mercury determination by CV-AFS. Journal of Hazardous Materials, 161(2-3), 1399-1403. https://doi.org/10.1016/j.jhazmat.2008.04.129

Bergés, T. M. E., Fernando, M. F, J., Torres, R, Y., Galván, M, F., \& Páez, O, F. (2015). Mercury and selenium in tissues and stomach contents of the migratory sailfish, Istiophorus platypterus, from the Eastern Pacific: Concentration, biomagnification, and dietary intake. Marine Pollution Bulletin, 101(1), 349-358. https://doi.org/ 10.1016/j.marpolbul.2015.10.021

Chaves, R. I. (2016). Metodologías analíticas utilizadas actualmente para la determinación de mercurio en músculo de pescado. Pensamiento Actual, 16(26), 113. https://doi.org/10.15517/pa.v16i26.25187

Comisión del Codex Alimentarius. (2018). Informe de la 12a. reunión del Comité del Codex sobre Contaminantes de los Alimentos. 88. Retrieved from http://www.fao. org/fao-who-codexalimentarius/sh-proxy/en/? Ink=1\&url=https \% 253A\%252F\%252Fworkspace.fao.org\%252Fsites\%252Fcodex\%252FMeetings\%2 52FCX-735-12\%252FREPORT\%252520\%2528FINAL\%2529\%252FREP18 -CFs.pdf

De Carvalho, G. G. A., Feres, M. A., Ferreira, J. R., \& Kennedy, V. H. (2010). Total and inorganic mercury determination in fish tissue by flow injection cold vapour atomic fluorescence spectrometry. International Journal of Environmental Analytical Chemistry, 90(9), 686-696. https://doi.org/10.1080/03067310902871729

EPA. (2000). Fish Sampling and Analysis. Guidance for Assessing Chemical Contaminant Data for Use in Fish Advisories, 1(4305), 485.

González, E. M., Bodas, P, A., Guillén, P, J. J., Rubio, H, M. Á., Ordóñez, I, J. M., Trasobares, I, E. M., Calle, P, A. (2014). Exposición al metilmercurio en la población general; toxicocinética; diferencias según el sexo, Factores nutricionales y genéticos. Nutricion Hospitalaria, 30(5), 969-988. https://doi.org/10.3305/nh.2014. 30.5.7727 
Gonzalez, E. M., Bodas, P, A., Martinez, G, M. J., Trasobares, I, E. Bermejo, B. M. P., Ordonez, I, J. M., Calle, P, A. (2015). Metilmercurio: Recomendaciones existentes; métodos de análisis e interpretación de resultados; evaluación económica. Nutricion Hospitalaria, 31(1), 1-15. https://doi.org/10.3305/nh.2015.31.1.8316

Krishna, M. V. B., Ranjit, M., Karunasagar, D., \& Arunachalam, J. (2005). A rapid ultrasound-assisted thiourea extraction method for the determination of inorganic and methyl mercury in biological and environmental samples by CVAAS. Talanta, 67(1), 70-80. https://doi.org/10.1016/j.talanta.2005.02.007

Moraes, P. M., Santos, F. A., Cavecci, B., Padilha, C. C. F., Vieira, J. C. S., Roldan, P. S., \& Padilha, P. D. M. (2013). GFAAS determination of mercury in muscle samples of fish from Amazon, Brazil. Food Chemistry, 141(3), 2614-2617. https://doi.org/ 10.1016/j.foodchem.2013.05.008

Müller, E. I., Mesko, M. F., Moraes, D. P., Korn, M. das G. A., \& Flores, É. M. M. (2014). Wet Digestion Using Microwave Heating. In Microwave-Assisted Sample Preparation for Trace Element Determination. https://doi.org/10.1016/B978-0-44459420-4.00004-0

Ochoa, C. (2015). Muestreo no probabilístico: muestreo por conveniencia. Recuperado en: https://www.netquest.com/blog/es/blog/es/muestreo-por-conveniencia

Pohl, P., Zapata, J. I., Voges, E., Bings, N. H., \& Broekaert, J. A. C. (2008). Comparison of the cold vapor generation using $\mathrm{NaBH} 4$ and $\mathrm{SnCl} 2$ as reducing agents and atomic emission spectrometry for the determination of $\mathrm{Hg}$ with a microstrip microwave induced argon plasma exiting from the wafer. Microchimica Acta, 161(1-2), 175-184. https://doi.org/10.1007/s00604-007-0887-8

Reyes, Y, C., Vergara, I. Torres O, E., Díaz, M, \& González, E, E. (2016). Contaminación por metales pesados: implicaciones en salud, ambiente y seguridad alimentaria. Recuperado en: https://sci-hub.tf/https://dialnet.unirioja.es/servlet/articulo?codigo $=6096110$

Rezende, P. S., Silva, N. C., Moura, W. D., \& Windmöller, C. C. (2018). Quantification and speciation of mercury in streams and rivers sediment samples from Paracatu, MG, Brazil, using a direct mercury analyzer $($. Microchemical Journal, 140(April), 199-206. https://doi.org/10.1016/j.microc.2018.04.006 
Segura, G. F. F., Ortega, M. L. C., \& Olaya, N, C. W. (2011). Relación longitud-peso de la Cachana (Cynopotamus atratoensis) en la ciénaga Grande de Lorica, Colombia. Acta Biologica Colombiana, 16(1), 77-86.

Shah, A. Q., Kazi, T. G., Baig, J. A., Afridi, H. I., \& Arain, M. B. (2012). Simultaneously determination of methyl and inorganic mercury in fish species by cold vapour generation atomic absorption spectrometry. Food Chemistry, 134(4), 2345-2349. https://doi.org/10.1016/j.foodchem.2012.03.109 